\title{
BAS MULTI-ROBOT FORMATION COORDINATION CONTROL FOR COLLECTIVE MISSIONS
}

\author{
Elżbieta M. Jarzębowska
}

Warsaw University of Technology, Institute of Aeronautics and Applied Mechanics, 00-665 Warsaw, Nowowiejska 24 St., Poland, e-mail:elajarz@meil.pw.edu.pl

\begin{abstract}
The paper presents a study of a mobile multi-robot formation (MRF) control problem. The robots are nonholonomic car-like vehicles and perform their mission while coordinating the formation shape when needed. The MRF control problem consists then of tracking reshaping a formation. Control design is based upon the centralized strategy assuming that information from a leader robot can be distributed to formation members. The main motivations for the work are that control strategies designed for single robots when applied to formations become complex and grow in terms of computational time, many of new control algorithms are dedicated to different formation coordination schemes and many of them were never verified experimentally. Two control levels are considered, i.e. kinematics and dynamics. Kinematics controllers applied are the input-output linearization and the Samson algorithm. They are simple in implementation and proved good performance in controlling single car-like robots. We examine them with regard to their potential applications for MRF. The dynamics controllers are based upon the generalized programmed motion equations method used successfully for a leader-follower control strategy for a fixed shape formation. Results of simulation studies, comparisons and discussions of implemented control algorithms are presented.
\end{abstract}

\section{Introduction}

The paper presents a study of a mobile multirobot formation (MRF) control problem. The robots are nonholonomic car-like vehicles and perform their mission while coordinating the formation shape when needed. The MRF control problem consists then of performing a mission and being able to reshape the formation. Control design is based upon the centralized strategy assuming that information from robot sensors enables determination of instantaneous relative position of each robot in the workspace, for example, by means of cameras that watch and monitor the entire environment. Also, a decentralized formation control scenario is studied when each member of the formation has information of its neighbors and not of the other robots in the formation. Altogether, three mission scenarios are presented using kinematics and dynamics based controllers. Problems of controlling and coordinating an autonomous MRF gain popularity due to number of benefits of coordinated collective missions in comparison with a single robot mission or a human activity, especially in a hazardous environment or when a task is distributed. Popularity of the MRF also comes from their numerous applications, e.g. transportation of payloads, reconnaissance, surveillance and rescue missions, cleaning and inspections in hazardous environment and many more $[7,8,9]$. In some situations, formation maintaining is not enough and a MRF may need to change its shape to conform a mission. 
Different approaches to MRF modeling, coordination and control are presented in the literature. Usually, members of a MRF are modeled as kinematic or dynamic car-like mobile vehicles or as particle agents that form a swarm equipped with various sensing possibilities [4,8]. There are three major approaches to formation control, i.e. behaviorbased, leader-follower and a virtual structure formation control. In the behavior-based formation control [6], a desired set of behaviors is implemented to individual robots. By grading the behaviors, the overall behavior of a robot is specified. In the leaderfollower control strategy [2,5], followers follow the selected leaders and they position themselves relative to them. In the virtual structure control [1], a formation is considered as a single structure and desired motions are assigned to it. The formation in the virtual structure approach is rigid such that geometric relationships between robots have to be fixed during motion. Thus, this formation cannot reshape. Formation reshaping control strategies were implemented in some studies together with the leader-follower approach. For example, in [2] a framework for cooperative control of a group of mobile robots that allows building a complex system from simple controllers is described. The switching between simple decentralized controllers allows formation reshaping while following a leader or performing a task. In [3] nonlinear control and the graph theory approach are used to study the formation control of mobile robots. The formation reshaping can be achieved by adding or deleting edges in the formation graph.

One of motivations for the presented work is that the problems with the control strategies in [2], [3] and also in other works are that their level of computational complexity grows with the increase of the number of robots in the formation. Also, new control algorithms are generated for different formation coordination schemes. They often suffer from complexity and often cannot be reused for other MRF when different tasks are specified. Also, many of them were never verified experimentally. In this work, a MRF coordination control as well as control of performing a desired task is designed using existing controllers at the kinematics and dynamics levels. The kinematics controllers selected to the study are the input-output linearization and the Samson algorithm. They are simple in implementation and proved good performance in controlling single car-like robots. We examine them with regard to their potential applications for MRF. The dynamics controllers are based upon the generalized programmed motion equations method used successfully for a leader-follower control strategy for a fixed shape formation [5]. The other motivation is to study formation reshaping scenarios when a change of a leader is needed. It may happen though that a leader robot fails during a mission and another robot is to take its role. Three mission scenarios are presented for reshaping the MRF and changing a leader.

Results of simulation studies as well as comparisons and discussions of implemented control algorithms, at the kinematics and dynamics levels, are presented in this work.

\section{Leader - follower formation control algorithms applied at kinematics and dynamics levels}

There are quite efficient and easy implementable control algorithms designed for a single robot and two of them were verified if they are applicable to MRF coordination as well as for performing desired tasks. The formation consists of Pioneer 3DX two wheeled robots, whose kinematics is equivalent to this of a unicycle, see Fig. 1.

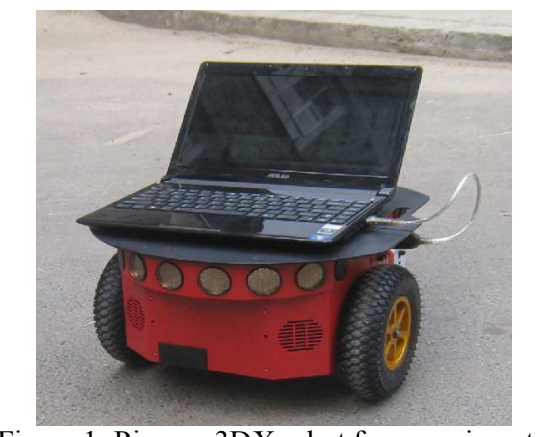

Figure 1. Pioneer 3DX robot for experiments

The first examined algorithm, at the kinematics level, is the input-output dynamics linearization, which provides full linearization, i.e. the resulting kinematic control model has the form

$$
\begin{aligned}
& \ddot{z}_{1}=u_{1}, \\
& \ddot{z}_{2}=u_{2},
\end{aligned}
$$

where $z_{i}$ are new states and $u_{i}$ are new inputs, e.g. PD inputs of the form

$$
\begin{aligned}
& u_{1}=\ddot{x}_{d}(t)+k_{d}\left(\dot{x}_{d}(t)-\dot{x}\right)+k_{p}\left(x_{d}(t)-x\right), \\
& u_{2}=\ddot{y}_{d}(t)+k_{d}\left(\dot{y}_{d}(t)-\dot{y}\right)+k_{p}\left(y_{d}(t)-y\right),
\end{aligned}
$$

Advantages of the input-output dynamics linearization are the exponential convergence, easy numerical and real robot implementation. A disadvantage is a structural singularity of the nonlinear state vector transformation, which requires a non-zero vehicle velocity.

The second examined algorithm is the Samson tracking control algorithm, which is based upon an 
error dynamics, which is obtained from the robot kinematics $A(q) \dot{q}=0$ by differentiating tracking errors determined in the robot frame, i.e.

$$
\left[\begin{array}{l}
x_{e} \\
y_{e} \\
\varphi_{e}
\end{array}\right]=\left[\begin{array}{ccc}
\cos \varphi & \sin \varphi & 0 \\
-\sin \varphi & \cos \varphi & 0 \\
0 & 0 & 1
\end{array}\right]\left[\begin{array}{l}
x_{d}-x \\
y_{d}-y \\
\varphi_{d}-\varphi
\end{array}\right]
$$

Inserting $\dot{x}_{d}=V_{d} \cos \varphi_{d}, \dot{y}_{d}=V_{d} \sin \varphi_{d}, \dot{\varphi}_{d}=\omega_{d}$ to $(2)$

along a desired trajectory one gets

$$
\left[\begin{array}{c}
\dot{x}_{e} \\
\dot{y}_{e} \\
\dot{\varphi}_{e}
\end{array}\right]=\left[\begin{array}{ccc}
0 & \omega & 0 \\
-\omega & 0 & 0 \\
0 & 0 & 0
\end{array}\right]\left[\begin{array}{c}
x_{e} \\
y_{e} \\
\varphi_{e}
\end{array}\right]+\left[\begin{array}{c}
V_{d} \cos \varphi_{e}-V \\
V_{d} \sin \varphi_{e} \\
\omega_{d}-\omega
\end{array}\right] \text {. }
$$

Transforming the original inputs to

$$
V=V_{d} \cos \varphi_{e}-u_{1}, \quad \omega=\omega_{d}-u_{2},
$$

yields

$$
\left[\begin{array}{c}
\dot{x}_{e} \\
\dot{y}_{e} \\
\dot{\varphi}_{e}
\end{array}\right]=\left[\begin{array}{ccc}
0 & \omega_{d} & 0 \\
-\omega_{d} & 0 & 0 \\
0 & 0 & 0
\end{array}\right]\left[\begin{array}{l}
x_{e} \\
y_{e} \\
\varphi_{e}
\end{array}\right]+\left[\begin{array}{c}
0 \\
\sin \varphi_{e} \\
0
\end{array}\right] V_{d}+\left[\begin{array}{ll}
1 & 0 \\
0 & 0 \\
0 & 1
\end{array}\right]\left[\begin{array}{l}
u_{1} \\
u_{2}
\end{array}\right] .
$$

New control inputs are defined as

$$
u_{1}=-k_{1} x_{e}, \quad u_{2}=-k_{2} \operatorname{sgn}\left[V_{d}(t)\right] y_{e}-k_{3} \varphi_{e},
$$

with $k_{1}, k_{2}$ and $k_{3}$ being control gains.

The Samson algorithm is not easy for numerical implementation due to control inputs $u_{i}$ that have no physical interpretation. Backward transformation is needed to assess real input magnitudes and one needs to transform $x, y$ and a heading angle $\varphi$ to their errors and back, what may affect computing initialization. Also, linear and angular velocities directly resulting from reference motion need to be calculated.

A dynamics control model for a single robot is

$$
\begin{gathered}
M(q) \ddot{q}+C(q, \dot{q}) \dot{q}+D(q)=E(q) \tau, \\
B_{1}(q) \dot{q}=0 .
\end{gathered}
$$

Applying static state feedback linearization yields

$$
\begin{gathered}
\dot{x}_{1}=G\left(x_{1}\right) x_{2}, \\
\dot{x}_{2}=u,
\end{gathered}
$$

The model (8) is taken for simulations for a MRF controlling a collective task mission.

\section{Simulation case studies and experiments}

Three simulation case studies and one experiment on a Pioneer robot are presented. The first two are for following a leader and an MRF reshaping during a mission. The third one is dynamics based control. The four robot formation starts from positions shown in fig. 2. A desired motion is along a trajectory of a shape $x_{d}(t)=\sin (0.1 t), \quad y_{d}(t)=\sin (0.1 t) \quad$ The followers start their motions every 6 seconds.

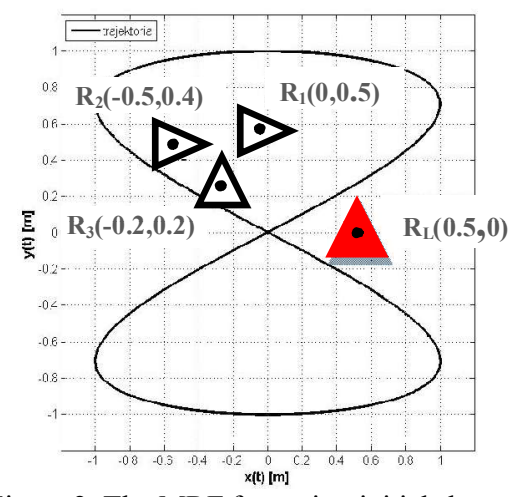

Figure 2. The MRF formation initial shape.

The simulation results, when robots follow a leader, are shown in fig. 3 for the input-output dynamics linearization and the Samson algorithm. In fig. 4 the analogous results are shown but this time a leader is a preceding robot. Notice the way robot 3 follows robot 2: it does the same ,zig-zag" motion; the Samson algorithm relies upon the heading angle and angular velocity of the preceding robot. Also, robots controlled by the Samson algorithm reach about 3 times bigger linear velocities than controlled by the input-output linearization. This is due to control gains but they are adjusted to get some reasonable the convergence rate. Differences between angular velocities are smaller; 2 times bigger for the robot 3 comparing to the input-output linearization.

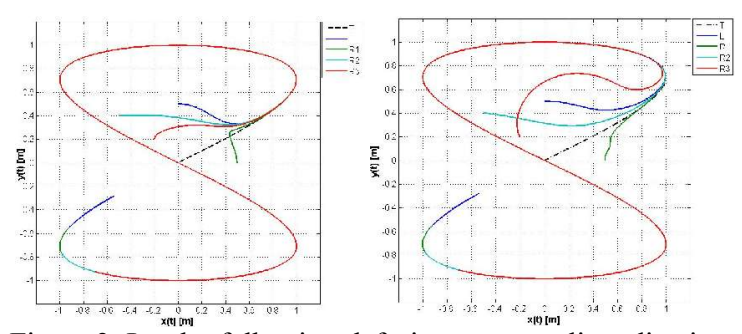

Figure 3. Leader following: left: input-output linearlization, right: Samson algorithm.
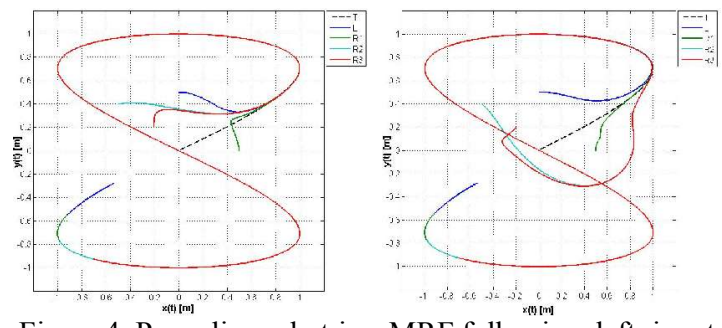

Figure 4. Preceding robot in a MRF following: left: inputoutput linearlization, right: Samson algorithm.

The reshaping scenario for the MRF is presented in fig. 5. The leader starts its motion, then the followers reach the desired trajectory, and the 
command about the reshaping is given - form a triangle and continue following the leader.

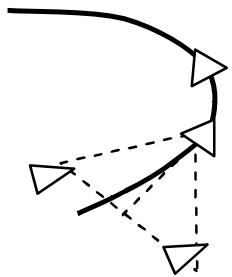

Figure 5. The reshaping scenario for the MRF.

Trajectory tracking results are shown in Fig. 6 for both kinematics based controllers.

The dynamics controller is based upon (7)-(8) and it is the Repetitive Control Law controller for a "zigzag" reconnaissance mission. The simulation results for a sparse MRF are shown in Fig. 7.

An experiment with the application of the inputoutput linearizing controller was performed on a Pioneer 3DX robot (Fig. 1). The Pioneer robot is quite slow and it follows a desired trajectory quite well. It required however, completely different selection of control gains comparing to the ones adjusted in simulations studies. Also, tracking errors, a simulated one (in red) and a real one (in blue) are much different. This is of course a result of cumulating errors from all delays in a real robot software and hardware, and lacking dynamics.
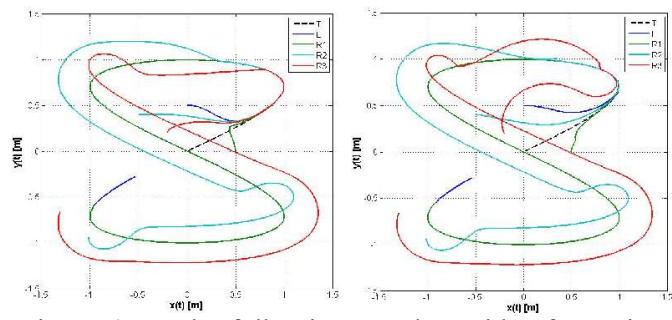

Figure 6. Leader following together with a formation reshaping: left: input-output linearlization, right: Samson algorithm.

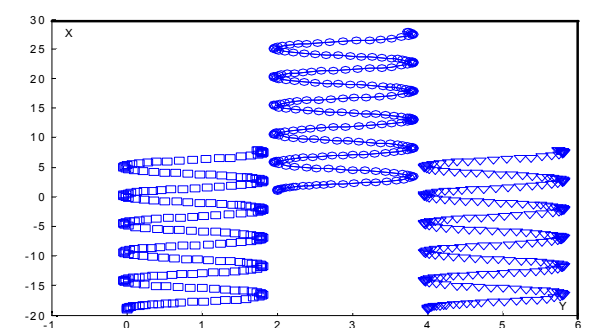

Figure 7. RCL controller for tracking a "zig-zag" reconnaissance mission for MRF.

Experimental results (in red) of a desired trajectory (in blue) tracking are shown in Fig. 8.

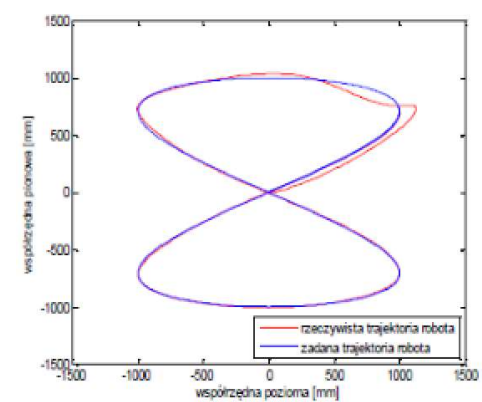

Figure 8 . Trajectory tracking by Pioneer 3DX (in red).

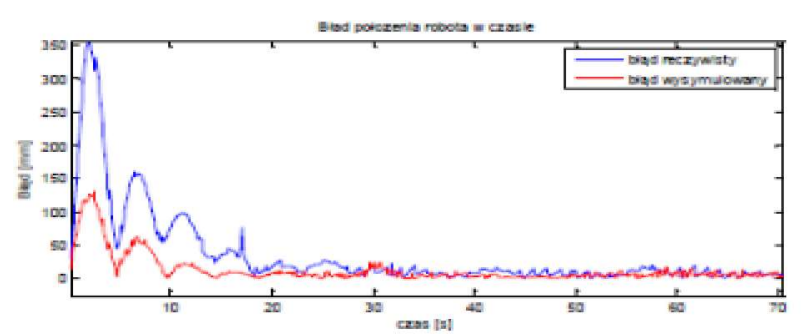

Figure 9. Position tracking errors: simulation - red, experiment - blue.

\section{Conclusions}

There are not many works verifying control algorithms for their further implementations. There are not many simulation studies for the control algorithms with regard of their applications to MRF. The future work is oriented to implementation of the algorithms to Pioneer robot.

\section{References}

1. Beard R.W., Lawton J., Hadaegh F.Y.: Coordination Architecture for Spacecraft Formation Control. IEEE Trans. Control Syst. Techn., vol. 9, no. 6, Nov. 2001, pp. 777-790.

2. Das A.K., Fierro R., Kumar V., Ostrowski J.P., Spletzer J., and Taylor C.J.: A Vision -Based Formation Control Framework. IEEE Trans. Robot. Automat., vol. 18, no. 5, 2002, pp. 813825.

3. Desai J.P., Kumar V., Ostrowski P.: Modeling and Control of Formations of Nonholonomic Mobile Robots. IEEE Trans. Robot. Automat., vol.17, Dec. 2001, pp. 905-908.

4. Hou S.P., Cheah C.C., Slotine J.J.E.: Dynamic Region Following Formation Control for a Swarm of Robots, 2009 IEEE Int. Conf. Robot. Automat., Kobe, Japan, May 12-17, 2009, pp. 1929-1934.

5. Jarzębowska E.: Leader-Follower Tracking Control Design for Task-Based Missions. Int. J. 
Vehicle Autonomous Syst., Vol. 9, No. 3/4, 2011, pp. 203-217.

6. Lawton J.R., Beard R.W., Young B.J.: A Decentralized Approach to Formation Maneuvers. IEEE Trans. Robot. Automat., vol. 19, no. 6, pp. 933-941, Dec. 2003.

7. Mas I., Li S., Acain J., Kitts C.: Entrapment/Escorting and Patrolling Missions in Multi-Robot Cluster Space Control. Proc. 2009 IEEE Int. Conf. Intelligent Robots and Systems, St. Louis, USA, October 2009, pp. 5855-5861.
8. Raghuwaiya K., Singh S., Sharma B., Vanualailai J.: Formation Types of a Flock of 1 Trailer Mobile Robots. 7th IMT-GT Int. Conf. Mathematics, Statistics and its Applications, 2011, pp. 368-382.

9. Raghuwaiya K., Singh S., Vanualailai J.: Formation Control of Mobile Robots. World Academy of Science, Engineering and Technology 60, 2011, pp. $762-767$. 